\title{
Annexin A6 is critical to maintain glucose homeostasis and survival during liver regeneration in mice
}

Anna Alvarez-Guaita ${ }^{1,2,+}$, Patricia Blanco-Muñoz ${ }^{1,3,+}$, Elsa Meneses-Salas ${ }^{1,3}$, Mohamed Wahba ${ }^{4}$, Abigail H. Pollock ${ }^{5}$, Jaimy Jose ${ }^{4}$, Mercedes Casado ${ }^{6}$, Marta Bosch ${ }^{3}$, Rafael Artuch ${ }^{6}$, Katharina Gaus $^{5}$, Albert Lu ${ }^{7}$, Albert Pol ${ }^{3,8}$, Francesc Tebar ${ }^{1,3}$, Stephen E. Moss ${ }^{9}$, Thomas Grewal ${ }^{4, *}$, Carlos Enrich ${ }^{1,3, *}$ and Carles Rentero ${ }^{1,3, *}$

${ }^{1}$ Departament de Biomedicina, Unitat de Biologia Cel·lular, Facultat de Medicina i Ciències de la Salut, Universitat de Barcelona, 08036 Barcelona, Spain.

${ }^{2}$ Currently at Institute of Metabolic Science, University of Cambridge, Cambridge CB2 0QQ, UK.

${ }^{3}$ Centre de Recerca Biomèdica CELLEX, Institut d'Investigacions Biomèdiques August Pi i Sunyer (IDIBAPS), 08036 Barcelona, Spain.

${ }^{4}$ School of Pharmacy, Faculty of Medicine and Health, University of Sydney, Sydney, NSW 2006, Australia.

5 Center for Vascular Research, The University of New South Wales, Sydney, NSW, 2052, Australia.

${ }^{6}$ Clinical Biochemistry Department, Institut de Recerca Sant Joan de Déu and CIBERER, Barcelona, Spain.

${ }^{7}$ Department of Biochemistry, Stanford University School of Medicine, Stanford, CA, 94305 United States

${ }^{8}$ Institució Catalana de Recerca i Estudis Avançats (ICREA), 08010 Barcelona, Spain.

${ }^{9}$ Institute of Ophthalmology, University College of London, 11-43 Bath Street, London EC1V 9EL, UK.

${ }^{+}$Both authors contributed equally.

* Corresponding authors:

Carles Rentero (carles.rentero@ub.edu)

Dept. de Biomedicina, Unitat de Biologia Cel·lular, Facultat de Medicina i Ciències de la Salut, Universitat de Barcelona.

Carrer Casanova, 143

08036 Barcelona, Spain.

Phone number: +34 2275400 ext. 3358

FAX number: +34 934021907

Carlos Enrich (enrich@ub.edu)

Dept. de Biomedicina, Unitat de Biologia Cel·lular, Facultat de Medicina i Ciències de la Salut, Universitat de Barcelona.

Carrer Casanova, 143

08036 Barcelona, Spain.

Phone number: +34 934021908

FAX number: +34 934021907 
Thomas Grewal (thomas.grewal@sydney.edu.au)

School of Pharmacy, Faculty of Medicine and Health, The University of Sydney.

Rm N518, Pharmacy and Bank Building A15

2006 Sydney (NSW), Australia.

Phone number: +61293518496

FAX number: +61293514391

Keywords: Liver regeneration; Annexin A6; Partial hepatectomy; Gluconeogenesis; Alanine; SNAT4

Conflict of Interest Statement: The authors declare no competing interests.

Financial Support Statement: This research received funding from grants BFU2015-66785-P, Consolider-Ingenio (CSD2009-00016 and BFU2016-81912-REDC) from the Ministerio de Economía y Competitividad (Spain), and PI042182 from Fundació La Marató de TV3 (Spain) to C.E. T.G. is supported by the University of Sydney (U7007, U7042, U7113, RY253), Sydney, Australia. 


\section{ABSTRACT}

Background \& Aims. Liver regeneration requires the organized and sequential activation of events that lead to the restoration of hepatic mass. During this process, other vital liver functions need to be preserved, such as the maintenance of blood glucose homeostasis, balancing the degradation of hepatic glycogen stores and gluconeogenesis (GNG). Under metabolic stress, alanine is the main hepatic gluconeogenic substrate, and its availability is the rate-limiting step in this pathway. The $\mathrm{Na}^{+}$-coupled neutral amino acid transporters (SNAT) 2 and 4 are believed to facilitate hepatic alanine uptake. In previous studies we demonstrated that a member of the $\mathrm{Ca}^{2+}{ }_{-}$ dependent phospholipid binding annexins, Annexin A6 (AnxA6), regulates membrane trafficking along endo- and exocytic pathways. Yet, although AnxA6 is abundantly expressed in the liver, its function in hepatic physiology remains unknown. In this study, we investigated the potential contribution of AnxA6 in liver regeneration.

Methods. Utilizing AnxA6 knock-out mice $\left(\mathrm{AnxA6}^{-/-}\right)$, we challenged liver function after partial hepatectomy (PHx) inducing acute proliferative and metabolic stress. Biochemical and immunofluorescent approaches were employed to dissect AnxA6 ${ }^{-/-}$mice liver proliferation and energetic metabolism.

$\underline{\text { Results. Most strikingly, AnxA6 }}{ }^{--}$mice exhibited low survival after PHx. This was associated with an irreversible and progressive drop of blood glucose levels. While exogenous glucose administration or restoration of hepatic AnxA6 expression rescued AnxA6 ${ }^{-/-}$mice survival after PHx, the sustained hypoglycaemia in partially hepatectomized AnxA6 ${ }^{-/}$mice was the consequence of an impaired alanine-dependent GNG in AnxA6 $6^{-/-}$hepatocytes. Mechanistically, cytoplasmic SNAT4 failed to recycle to the sinusoidal plasma membrane of AnxA6 ${ }^{-/}$ hepatocytes $48 \mathrm{~h}$ after PHx, impairing alanine uptake and consequently, glucose production.

Conclusion: We conclude that the lack of AnxA6 compromises alanine-dependent GNG and liver regeneration in mice. 


\section{INTRODUCTION}

The liver is a multifunctional organ that has a central role in metabolic pathways, blood detoxification and the maintenance of plasma protein levels. The preservation of these metabolic and detoxifying capacities forces the liver to establish a unique and rapid organ regeneration program after an insult or partial loss of its mass meanwhile maintaining its essential functions (1). Pathways leading to the completion of regeneration are evolutionary conserved and mostly redundant, and thus the phenotype of the majority of genetically modified mice models that have been examined show a delay rather than a complete abrogation of hepatic regeneration (1). The most common approach to study liver regeneration is the partial hepatectomy (PHx) model, which triggers the acute phase response, a rapid, complex and pleiotropic response that induces entry into the cell cycle (G0 - G1 phase transition) to then accomplish S phase prior to the onset of hepatocellular proliferation to restore the original hepatic mass per hyperplasia $(1,2)$.

Metabolically, the removal of two-thirds of hepatic mass through PHx in mice triggers a rapid fall in blood glucose levels due to a dramatic reduction of glycogen stores and gluconeogenic capacity (3). Although this demonstrates that the remnant liver is initially unable to fully control the maintenance of glucose homeostasis, some metabolic adaptations such as suppression of liver glycolytic activity and induction of the hepatic gluconeogenic machinery occur soon after PHx to restore blood glucose levels. During liver regeneration, a shift of pyruvate metabolization from acetyl-CoA to the oxalacetic pathway is observed, enabling the de novo formation of glucose from pyruvate (4). In this context, alanine is the major gluconeogenic substrate to produce pyruvate, as also evidenced by the markedly increased incorporation of alanine carbons into plasma glucose (5).

In hepatocytes, alanine uptake is mainly facilitated by the ubiquitously expressed $\mathrm{Na}^{+}-$ coupled neutral amino acid transporter (SNAT) 2 and the liver (human, rat)- and muscle (rat)specific SNAT4, both system A transporters that are recruited to the plasma membrane under not well defined metabolic conditions $(6,7)$. Although a critical step, little is known about the regulation of the targeting of the SNAT proteins to the sinusoidal plasma membrane in the liver (8), as this represents the blood-facing surface containing the greatest concentration of hormone and growth factor receptors, as well as metabolites and amino acid transporters. Hence, the proper functioning of the sinusoidal plasma membrane and trafficking/exchange of receptors and 
transporters with the underlying receptor-recycling endocytic compartment is crucial for the reception and/or transduction of the early signals that trigger hepatic cell activation (9).

Annexin A6 (AnxA6), a member of the annexin family, is a $\mathrm{Ca}^{2+}$-dependent phospholipid-binding protein involved in the regulation of a plethora of cellular functions including membrane trafficking (10), cholesterol homeostasis $(11,12)$, signalling (13), as well as cortical actin-cytoskeleton and plasma membrane microdomain re-arrangements (14). Despite these multiple functions in various cellular locations, AnxA6 is mainly found at the plasma membrane and endocytic compartments of rodent hepatocytes (9), representing approximately $0.25 \%$ of total rat hepatic protein (15). AnxA6 knock-out (AnxA6 ${ }^{-/}$) mice appear normal (16), with the only exceptions being AnxA6 ${ }^{-/}$cardiomyocytes exhibiting higher contractility and accelerated removal of diastolic $\mathrm{Ca}^{2+}$ from the cytoplasm (17) and impaired adiponectin secretion of AnxA6 $6^{-/}$adipocytes (18). Moreover, upon high-fat diet feeding, AnxA6 ${ }^{-/-}$mice displayed reduced weight gain and adiposity, and an inability to induce insulin-dependent downregulation of GNG (19). The latter observations pointed at metabolically relevant hepatic AnxA6 functions, but its precise role in liver physiology has yet remained elusive and might only be revealed during metabolic adaptions required to overcome stress conditions.

In the present study we identify that loss of AnxA6 dramatically reduced mice survival after PHx, without affecting the acute phase response and the cell cycle progression through the regeneration program. These findings were associated with a delay in the lipid storage kinetics and prolonged hypoglycaemia after PHx. Markedly, the exogenous administration of glucose restored survival in AnxA6 ${ }^{-/-}$mice after PHx, highlighting an energetic metabolic defect in the liver upon AnxA6 depletion in vivo. Further analysis showed impaired alanine-dependent GNG and alanine uptake in AnxA6 ${ }^{-/-}$primary hepatocytes. Moreover, this coincided with a compromised shuttling of the liver-specific alanine transporter SNAT4 between the sinusoidal plasma membrane and cytoplasmic structures in AnxA6 ${ }^{--}$mice, which appeared the underlying cause for the strongly restrained uptake and utilization of alanine for GNG after PHx. This is the first study that recognizes a critical role for hepatic AnxA6 in vivo, with striking consequences for the regulation of the energetic metabolism during liver regeneration in mice. 


\section{MATERIALS AND METHODS}

\section{Animals}

Eight to twelve week-old C57B16/J wild type and AnxA6 ${ }^{-/-}$male mice were maintained in a $12 \mathrm{~h}$ light/dark cycle, allowed food and water ad libitum. Every effort was made to minimize animal suffering and to use the minimum number of animals per group and experiment. All the animal care and experimental procedures were approved by the Local Ethical Committee of the University of Barcelona following European (2010/63/UE) and Spanish (RD 53/2013) regulations for the care and use of laboratory animals.

\section{Partial hepatectomy}

A 70\% PHx was performed under 2\% isoflurane anaesthesia as described (20). As control, sham operations were performed but livers were not removed. Mice were killed and liver samples were harvested at the different time points specified in each experiment. The hepatic index (liver weight/body weight) was monitored for each mouse over 7 days.

\section{Liver section immunohistochemistry and determination of steatosis}

Liver tissue was formaldehyde-fixed and paraffin-embedded in blocks. $5 \mu \mathrm{m}$ thick liver sections were de-paraffined, rehydrated, then heated to $100^{\circ} \mathrm{C}$ for $5 \mathrm{~min}$ in citrate buffer (pH 6.0) for antigen retrieval. Immunohistochemistry for proliferating cell nuclear antigen (PCNA; Santa Cruz, sc-25280) was performed using the ImmunoCruz Mouse ABC Staining System (Santa Cruz) according to the manufacturer's instructions. Liver sections were stained for haematoxylin/eosin (HE) and steatosis was double-blind quantified from low (0) to high (5) steatosis.

For Ki67 immunostaining, liver tissue was formaldehyde-fixed for $24 \mathrm{~h}$ and $30 \%$ sucrose cryopreserved for $24 \mathrm{~h}$. The sample was embedded in OCT (Tissue-Tek) at $-80^{\circ} \mathrm{C}$. Liver sections (10 $\mu \mathrm{m}$ thick) were blocked with 1\% bovine serum albumin for $20 \mathrm{~min}$ and incubated with rabbit anti-Ki67 (Abcam, ab15580) and secondary antibody goat anti-rabbit-IgG-A647 (Life Technologies, A21244). The samples were mounted in Mowiol (Calbiochem). 


\section{Hepatic DNA synthesis}

$\left[{ }^{3} \mathrm{H}\right]$-thymidine incorporation into liver DNA was measured as described previously $(21) .\left[{ }^{3} \mathrm{H}\right]-$ thymidine (PerkinElmer) $(1 \mu \mathrm{Ci} / \mathrm{g}$ body weight) was injected intraperitoneally at different intervals after PHx. After $2 \mathrm{~h}$, the remnant liver $(300 \mathrm{mg}$ ) was excised and homogenized in 5\% trichloroacetic acid (TCA, Sigma) at $4^{\circ} \mathrm{C}$. After centrifugation at $16,800 \mathrm{~g}$ for $5 \mathrm{~min}$, the supernatant was discarded and the pellet resuspended in $1 \mathrm{ml} 5 \%$ TCA at $4^{\circ} \mathrm{C}$. This centrifugation step was repeated 3 times. Finally, the pellet was diluted in TCA and 1.5 N perchloric acid and incubated for $15 \mathrm{~min}$ at $90^{\circ} \mathrm{C}$, followed by centrifugation at $16,800 \mathrm{~g}$ for 5 min. The supernatant was collected and the amount of radioactivity was determined by liquid scintillation counting.

\section{Transmission electron microscopy (TEM)}

For TEM, livers were taken from mice following anaesthesia after intracardial perfusion with $2.5 \%$ glutaraldehyde in phosphate buffer. Liver samples were then fixed overnight in $2.5 \%$ glutaraldehyde and $4 \%$ paraformaldehyde. Small cubes of $1 \mathrm{~mm}^{3}$ were post-fixed in osmium tetroxide and embedded in Spurr (Sigma). TEM images were acquired from ultrathin sections using a JEOL-1010 electron microscope (JEOL USA) with a SC1000 ORIUS-CCD digital camera (Gatan).

\section{Alanine uptake assays}

Primary hepatocytes were isolated as described in Supplementary Material and Methods. To avoid interference with feedback mechanism, $3 \times 10^{6}$ cells $/ \mathrm{ml}$ in Krebs buffer were first incubated with $1 \mathrm{mM}$ amino-oxyacetic acid (Sigma), an inhibitor of alanine metabolism, for $10 \mathrm{~min}$ at $37^{\circ} \mathrm{C}$. Next, $1 \mu \mathrm{Ci}\left[{ }^{14} \mathrm{C}\right]$-L-alanine (PerkinElmer) was added and after 2, 5 and $10 \mathrm{~min}$, aliquots were taken. The reaction was stopped by addition of $1 \mathrm{ml}$ ice-cold Krebs albumin buffer and immediate centrifugation at $1,000 \mathrm{~g}$ for $5 \mathrm{sec}$. The supernatant was discarded and the cell pellets were resuspended in $1 \mathrm{ml}$ ice-cold Krebs buffer. This procedure was repeated twice, finally the radioactivity in the cell pellets was determined by liquid scintillation counting. 


\section{Statistics}

Data are shown as means \pm SEM. Statistical comparison of two groups was performed using a Student's $t$ test; analysis of mice survival was performed with a log-rank (Mantel-Cox) test; analysis of interaction was performed with a two-way analysis of variance (ANOVA) with $a d$ hoc Bonferroni post-test. Symbols represent $* p<0.05, * * p<0.01, * * * p<0.001$. Statistical analysis was performed in GraphPad Prism 8.

\section{RESULTS}

Impaired liver regeneration in AnxA6 $6^{-1-}$ mice

Despite AnxA6 being one of the most abundant hepatic proteins, its role in liver physiology is still unknown (9). Previous studies from our group and others analysing AnxA6 deficiency in C57B16/J mice $\left(\mathrm{AnxA6}^{-/}\right.$) did not reveal a phenotype that would suggest liver dysfunction $(16,18)$. In line with these findings, plasma levels of AST, ALAT, LDH; albumin and total protein, plasma lipid content (free cholesterol, TAG and free fatty acids); and HDL and LDL levels were all comparable in control and AnxA6 ${ }^{-/-}$mice (Supporting Fig. S1A-C).

To identify a possible role for AnxA6 in hepatic physiology, 2/3 partial hepatectomy (PHx) was performed in AnxA6 ${ }^{-/-}$mice and control littermates to induce both a proliferative and metabolic stress. Remarkably, and in striking contrast to the $95.7 \%$ survival rate of WT animals, AnxA6 deficiency was associated with high mortality and only $25.9 \%$ of AnxA6 $6^{-/}$mice survived $72 \mathrm{~h}$ after PHx (Fig. 1A). AnxA6 ${ }^{-/-}$mice recovered completely after a sham operation, excluding surgery and anaesthesia as a cause of mortality. The hepatic index (ratio between body and liver weight) was similar in both $\mathrm{AnxA6}^{-/-}$and WT mice until $48 \mathrm{~h}$ after liver resection (Fig. 1B). Plasma ALAT and AST levels appeared slightly elevated in AnxA6 ${ }^{-/-}$mice during the recovery after surgery. Although indicative of liver dysfunction, these findings were not significant (Supporting Fig. S1D-E). Hepatic AnxA6 protein levels in WT mice were slightly increased after PHx (Fig. 1C), though AnxA6 mRNA levels remained constant over this period (data not shown), indicating an increased protein stability of hepatic AnxA6 during liver regeneration.

Next we examined if restoration of hepatic AnxA6 expression could rescue survival of AnxA6 $6^{--}$mice after PHx. Therefore, we expressed recombinant AnxA6 using adeno-associated viral (rAAV) particles that contain the long terminal repeat of AAV2 in combination with 
envelop proteins of serotype 8, which enables liver-specific infection. Viral AnxA6 expression was driven by the human $\alpha-1$-antitrypsin promoter with regulatory sequences from the human albumin enhancer (see Supplementary Material and Methods for further details). Indeed, 15 days after infection of AnxA6 ${ }^{-/}$mice with rAAV-AnxA6, recombinant AnxA6 expression was only detectable in the liver, but not in other tissues, including lung and kidney (Fig. 1D). Notably, restoration of hepatic AnxA6 expression in AnxA6-deficient animals rescued survival of AnxA6/- mice after surgery in contrast to hepatic overexpression of recombinant GFP (red line versus orange line in Fig. 1E). Recombinant AnxA6 expression also restored the hepatic index 7 days after PHx in AnxA6 ${ }^{-/}$mice (Fig. 1F). Thus, restoration of liver-specific AnxA6 expression overcame physiological defects in $\mathrm{AnxA6} 6^{-/-}$mice that caused death after PHx.

We next examined several markers of cellular proliferation in the early stages of liver regeneration (22). The number of Ki67-positive hepatocytes (Fig. 2A), a cell cycle progression marker, was similar in both mice strains after PHx. In addition, the upregulation of cJun (Fig. 2B) and $c$ Fos (Fig. 2C) mRNA levels during the early regeneration process was comparable in WT and AnxA6-/- mice liver $1 \mathrm{~h}$ after liver resection (see Supplementary Material and Methods). Furthermore, mRNA upregulation of the S phase marker cyclin D1 (Ccnd1) (Fig. 2D) and nuclear PCNA staining (Fig. 2E), as well as the incorporation of radiolabelled thymidine into DNA (Fig. 2F), indicated a comparable DNA synthesis rate of WT and AnxA6 ${ }^{-/}$strains during the early post-PHx phase. Altogether this pointed at AnxA6 $6^{-/-}$hepatocytes progressing normally into the cell cycle in the early stages after PHx.

Hence, these results identified hepatic AnxA6 as critically contributing to mice survival during liver regeneration, resulting in high mortality of AnxA6 $6^{-/-}$mice after PHx. However, the loss of AnxA6 did not interfere with hepatocyte cell cycle progression required in the prereplicative phase of liver regeneration.

\section{Delay in steatosis associated with liver regeneration in AnxA6-- mice}

Steatosis is the next key step in liver regeneration (23), triggered within a few hours of liver resection and essential to effectively cover for an increased energy and structural lipid demand. Therefore, hepatic steatosis levels 12 and $36 \mathrm{~h}$ after PHx in AnxA6 ${ }^{-/}$and WT mice were compared using H\&E-stained liver sections (Fig. 3A). Double-blind quantification revealed a statistically significant delay in regeneration-induced steatosis in livers from AnxA6 ${ }^{-/}$mice 
(Fig. 3B), which correlated with notably $(\mathrm{p}<0.01)$ reduced hepatic TAG levels in AnxA6 $6^{-/-}$mice compared to WT mice (Fig. 3C). When analysing liver ultrastructure by electron microscopy (Fig. 3D), cytosolic glycogen stores (arrowheads) and few lipid droplets (*, LDs) were observed in both WT and AnxA6 ${ }^{--}$mice prior to liver resection $(\mathrm{t}=0)$. Although there was a delay in PHxinduced LD formation in AnxA6 ${ }^{-/}$animals $12 \mathrm{~h}$ post-PHx, LD numbers and size were comparable to WT 36-48 h post-hepatectomy. Levels of ketone bodies in the blood were similar in WT and AnxA6 ${ }^{-/-}$mice after PHx (Fig. 3E), indicating comparable lipid oxidation capacity of both strains. Hence, we conclude that the delayed commencement of steatosis observed in $\mathrm{AnxA6}^{-/-}$mice did not dramatically interfere with the onset of the liver regeneration program.

\section{Alterations in glucose metabolism after partial hepatectomy in AnxA6-1- mice}

Since $A n x A 6^{-/-}$mice displayed cell cycle progression comparable to control animals, we next analysed the energetic metabolism fate of AnxA6 ${ }^{-/-}$mice during liver regeneration. Initially we monitored blood glucose levels, which remained stable for approximately $1 \mathrm{~h}$ in WT mice after PHx, followed by hypoglycaemia 6-24 h post-PHx (Fig. 4A), as described previously (3). In WT animals, blood glucose levels started to rise thereafter, reaching and maintaining euglycemia levels at 36-48 h post-PHx. In striking contrast to the control animals, blood glucose levels in AnxA6 $6^{-/-}$mice dropped dramatically within $60 \mathrm{~min}$ after $\mathrm{PHx}$ to remain at hypoglycaemic levels even $72 \mathrm{~h}$ thereafter (Fig. 4A). Interestingly, the inability of AnxA6 ${ }^{-/}$mice to restore glucose homeostasis did not appear to involve the well-documented changes in insulin levels during PHx (24), with kinetics comparable to WT mice (Fig. 4B). Also, the ability to degrade liver glycogen stores was not compromised in AnxA6 ${ }^{-/-}$mice (Fig. 4C). In fact, glycogen degradation in AnxA6 /- was strongly increased over the course of the PHx compared to WT mice. Moreover, in line with the rescued survival of AnxA6 ${ }^{-/-}$mice after surgery upon viral-mediated restoration of hepatic AnxA6 expression (Fig. 1D-E), liver infection of these mice with AnxA6 expressing rAAV particles reduced the initial drop in blood glucose levels, and most strikingly, restored euglycemia $24 \mathrm{~h}$ after PHx comparable to WT mice (Supporting Fig. S2A).

Taken together, these results implicated a major defect in glucose homeostasis being responsible for the loss of survival after $\mathrm{PHx}$ in $\mathrm{AnxA6}^{-/-}$mice. 


\section{Glucose supplementation restores survival of AnxA6-- mice after PHx}

To reinforce the critical role of AnxA6 linking liver regeneration and glucose homeostasis, we explored the possibility of rescuing survival of AnxA6 $6^{-/}$mice after $\mathrm{PHx}$ providing glucose, as it is the predominant energy substrate during liver regeneration (25). Therefore, WT and AnxA6 ${ }^{-/}$mice were supplied ad libitum with $10 \%$ glucose in the drinking water 3 days prior liver resection and then throughout the regeneration period after surgery ( 7 days). Most outstandingly, AnxA6 ${ }^{-/}$mice supplemented with $10 \%$ glucose significantly recovered from $2 / 3 \mathrm{PHx}$, reaching survival rates similar to WT mice (Fig. 4D). These mice also recovered their hepatic index 7 days after PHx (Fig. 4E), indicating full liver regeneration capability. The improved survival of glucose-fed AnxA6 $6^{-/}$mice after liver resection correlated with restoration of glucose homeostasis (Fig. 4F), as blood glucose levels started to rise in glucose-supplemented AnxA6 ${ }^{-/}$mice $12 \mathrm{~h}$ after PHx, reaching euglycemia levels similar to WT $72 \mathrm{~h}$ post-PHx. Taken together, these results further strengthened AnxA6 to be a decisive factor in the hepatic glucose metabolism during liver regeneration.

\section{AnxA6 is essential for alanine-dependent hepatic gluconeogenesis}

The tricarboxylic acid (TCA) cycle provides carbons to fuel GNG and fatty acid synthesis during hepatic regeneration (5). Given the altered glucose homeostasis after liver resection in AnxA6 $^{-/}$mice (Fig. 4), we next analysed AnxA6-dependent hepatic GNG capability in vitro. Primary hepatocytes were isolated from livers of fasted ( $6 \mathrm{~h}$ to deplete their glycogen stores) WT and $A n x A 6^{-/ 2}$ mice, and GNG capacity was then measured as the ability to release glucose to the media in response to the incubation with pyruvate, glutamine or alanine (see Supplementary Material and Methods). No significant differences were observed in the secreted glucose levels when pyruvate or glutamine were provided as substrates (Fig. 5A). However, AnxA6 $6^{-/}$primary hepatocytes were completely unable to produce glucose when supplemented with alanine, strongly supporting AnxA6 being essential for alanine-driven GNG in hepatocytes. To analyse alanine availability for hepatic GNG during liver regeneration, we measured the amino acid content in both plasma and liver of WT and $\mathrm{AnxA6}^{-/-}$mice at 0, 12, 24 and $48 \mathrm{~h}$ after PHx (Fig. 5B-5E and Supplementary Fig. S3 and S4). Remarkably, plasma alanine levels remained unchanged during the first $24 \mathrm{~h}$ after PHx, and then increased at $48 \mathrm{~h}$ in WT mice, while AnxA6-

${ }^{\prime-}$ mice showed a much stronger increase (2.5-fold) of alanine plasma levels $24 \mathrm{~h}$ after PHx (Fig. 
5B). Hence, AnxA6 depletion in other tissues, in particular skeletal muscle, did not lead to defects in alanine release from extrahepatic tissues and sufficient amounts of alanine were available to the liver as a gluconeogenic substrate. In line with this, total plasma amino acid levels initialy increased after PHx in both WT and AnxA6 ${ }^{-/}$mice (Fig. 5C). Twenty-four hours after PHx uptake of plasma alanine (which represents $30 \%$ of total amino acids) decreased total plasma amino acid levels in WT mice, while remained high in AnxA6 ${ }^{-/}$mice. Interestingly, plasma branched-chain amino acids (BCAAs; Val, Leu and Ile) levels showed a peak $12 \mathrm{~h}$ after PHx in WT mice, which was not detected in the plasma of AnxA6 $6^{-/-}$mice (Supplementary Fig. S5A), suggesting BCAA catabolization for energy production in $A n x 6^{-/}$mice in the early stages of PHx. Alanine levels in the liver decreased $24 \mathrm{~h}$ after PHx in WT mice, probably because of its role to serve as a GNG substrate. However, hepatic alanine levels were restored 48 $\mathrm{h}$ after PHx, indicating active alanine uptake from the blood to overcome rate-limiting amounts in the GNG pathway (see Fig. 5D). In contrast, liver alanine levels did not recover $48 \mathrm{~h}$ after $\mathrm{PHx}$ in AnxA6 ${ }^{-/}$mice, suggesting regular alanine catabolism but a strongly compromised hepatic alanine uptake. These data correlated with the continuous decrease in total amino acid (Fig. 5E) and BCAA (Supplementary Fig. S5B) levels in the AnxA6 ${ }^{-/}$mice livers after PHx. Taken together, the inability of $A n x A 6^{-/}$hepatocytes to produce glucose from alanine (Fig. 5A) correlated with reduced availability of alanine for GNG after PHx, indicating that AnxA6 depletion interfered with alanine uptake in the liver after surgery.

To address aspects downstream of alanine uptake that might contribute to the AnxA6-/phenotype after PHx, we also analysed alanine deamination, the first step in hepatic alanine metabolization after PHx and essential for the formation of intermediates for the TCA cycle (4), and phosphoenolpyruvate carboxykinase (PEPCK) (Pckl) expression levels as the main control point for the regulation of GNG (4). Actually, hepatic Gptl (cytosolic glutamate-pyruvate transaminase, also termed ALAT1) levels (Fig. 6A) and ALAT activity (Fig. 6C) were comparable in both mouse strains during PHx. Yet, PHx-induced upregulation of Gpt2 (mitochondrial GPT, ALAT2; Fig. 6B) and Pckl observed in livers from WT mice (Fig. 6D) was absent in $\mathrm{AnxA6}^{-/}$livers, the latter indicating the lack of response to upregulated hepatic PEPCK levels to accommodate for increased amounts of alanine-derived pyruvate and carbon withdrawn from the TCA cycle to GNG after PHx. 
Altogether, these results indicated that hepatic GNG was strongly inhibited in AnxA6 $6^{-/}$ mice due to a dramatic impairment of alanine uptake in hepatocytes, while blood alanine levels and alanine deamination activity was not affected.

AnxA6 deficiency inhibits recycling of the alanine transporter SNAT4 to the hepatocyte plasma membrane after $\mathrm{PHx}$

Previous results revealed an incapacity of $A n x \mathrm{A6}^{-/-}$primary hepatocytes to produce glucose from alanine, while GNG competence (from glutamine and pyruvate), blood alanine availability and hepatic deamination activity was unaffected. Given that hepatic alanine levels failed to rise $48 \mathrm{~h}$ after PHx (Fig. 5D), we next analysed the uptake of radiolabelled alanine in WT and AnxA6 ${ }^{-1-}$ primary hepatocytes. Indeed, in contrast to the linear increase of alanine uptake in WT hepatocytes, AnxA6 ${ }^{-/}$hepatocytes completely lacked the ability to internalize radiolabelled alanine (Fig. 7A). Together with the upregulated levels of alanine in plasma, yet reduced liver alanine levels after $\mathrm{PHx}$, these findings pointed at a failure in hepatic uptake of circulating alanine as a major underlying cause for the impaired alanine-promoted GNG in the regenerating liver of $\mathrm{AnxA6}^{-/}$mice.

This prompted us to analyse the expression levels of hepatic alanine transporters, SNAT2 and SNAT4, by quantitative RT-PCR at different time points after PHx in WT and AnxA6 ${ }^{-/}$ mice (Fig. 7B-C). Interestingly, SNAT2 mRNA levels remained constant in both mice strains after PHx, while SNAT4 mRNA expression increased approximately 13-fold after liver resection in WT, but only 5-fold in AnxA6-/- mice. Likewise, although not significant, hepatic protein levels of SNAT4 were reduced by $20 \%$ in AnxA6 $6^{-/}$mice compared to WT $48 \mathrm{~h}$ after PHx (Fig. 7D), altogether implicating the lack of SNAT4 upregulation contributing to compromise alanine uptake and metabolization during liver regeneration of AnxA6-deficient animals.

In addition to the loss of stress-provoked upregulation of hepatic SNAT4 expression levels in AnxA6--- mice, we addressed if alterations in SNAT4 function could be affected in AnxA6 $6^{-/}$livers. It is well-known that the localization and trafficking of membrane proteins is intimately linked to their functionality. In this context, several transporters, including the glucose transporter 4, or integrins, are known to reside in the recycling endocytic compartment shuttling back-and-forth to the plasma membrane upon physiological alteractions and signals (26). Likewise, current cellular models have pointed towards metabolic changes to induce trafficking 
and delivery of amino acid transporters to the plasma membrane in order to increase amino acid availability $(6,27)$. Therefore, we next analysed the subcellular localization of SNAT4 during hepatic regeneration in WT and AnxA6-/- mice by fluorescence immunohistochemistry (Fig. 7E, quantified in 7F). Prior to liver resection surgery, SNAT4 immunostaining displayed an intracellular scattered location pattern both in WT and AnxA6 ${ }^{-/-}$hepatocytes. PHx triggered the shuttling of SNAT4 to the plasma membrane $(12 \mathrm{~h})$ and its recycling to cytoplasmic structures at the sub-sinusoidal region of hepatocytes $(24 \mathrm{~h})$. Most remarkably, $48 \mathrm{~h}$ after PHx, the SNAT4 transporter was translocated to the sinusoidal plasma membrane in WT mice, indicating that this change in SNAT4 location could provide a means to deliver increased amounts of blood alanine to hepatocytes in order to drive GNG. In AnxA6 $6^{-/-}$livers, and similar to WT hepatocytes, SNAT4 was initially located in the sinusoidal plasma membrane $12 \mathrm{~h}$ after $\mathrm{PHx}$, and returned to cytoplasmic structures $24 \mathrm{~h}$ after PHx (Fig. 7E, quantified in 7F). However, in striking contrast to the translocation of SNAT4 to the sinusoidal membrane in WT hepatocytes $48 \mathrm{~h}$ after PHx, SNAT4 remained in vesicular intracellular structures in AnxA6 $6^{-/-}$hepatocytes at this later time point after PHx. Hence, AnxA6 depletion critically interfered with the recycling of SNAT4 to the plasma membrane upon prolonged metabolic stress during liver regeneration. Indeed, viralmediated hepatic expression of AnxA6 in AnxA6 ${ }^{-/}$mice restored SNAT4 trafficking after surgery, further supporting that AnxA6 is enabling SNAT4 transport to the sinusoidal plasma membrane $48 \mathrm{~h}$ after surgery (see Supplementary Fig. S2B, quantified in S2C).

Hence, AnxA6 deficiency is associated with impaired SNAT4 recycling to the sinusoidal plasma membrane post-PHx, leading to defects in hepatic alanine metabolism that is required to drive GNG and maintain glucose homeostasis during liver regeneration.

\section{DISCUSSION}

In this study we demonstrate that AnxA6 is a critical factor contributing to liver regeneration. AnxA6 deficiency leads to a low survival rate after PHx and is associated with sustained and irreversible hypoglycaemia during the post-hepatectomy phase. AnxA6 is not necessary for regular cell cycle progression and proliferation of hepatocytes, but for maintaining the heavy energetic requirements after PHx. This striking phenotype of AnxA6-/- mice can be explained by the impaired recycling of SNAT4, the main alanine transporter during liver 
regeneration, to the hepatocyte sinusoidal plasma membrane. Given that the exogenous administration of glucose during liver regeneration restored AnxA6 ${ }^{-/-}$mice survival after PHx, we conclude that this novel role for AnxA6 in the control of SNAT4 membrane translocation is decisive to fuel alanine-dependent hepatic GNG during liver regeneration (Fig. 8).

The success of many treatments for chronic liver diseases, as well as for liver transplantation, relies on its regeneration efficiency. Hence, a better understanding of liver regeneration is clinically relevant, yet the regulation of this complex and pleiotropic process is still elusive due to the intricate spatiotemporal coordination of cytokines, growth factors, metabolic signalling pathways and extracellular matrix deposition that have been proven essential to re-establish the architecture and function of the liver (2). Intriguinly, while the majority of knock-out mouse models described to date display a delayed rather than complete abrogation of regeneration (1), the present study identifies AnxA6 not only as a novel, but also decisive factor crucial in the post-hepatectomy phase, a feature that is documented only for a rare number of genes $(23,28,29)$.

An early steatotic phase is a hallmark for hepatic regeneration, and fatty acids serve as energy source during the initial steps of regeneration $(30,31)$. Accordingly, several studies showed that a decrease in hepatic steatosis diminished liver regeneration $(23,32-35)$. In addition, despite some controversy $(36,37)$, glucose availability is another key factor during liver regeneration (38) and it was suggested that the hepatic energy status is vital for cell proliferation and survival after PHx $(30,38)$. Here, AnxA6 ${ }^{-/}$animals displayed a significant delay of LD formation that did not affect the kinetics of S phase entry. The high glucose demands of AnxA6 $6^{--}$mice, as evidenced by the fast reduction in blood glucose levels and high hepatic glycogen degradation rate, together with the normal hepatic capacity to perform betaoxidation in these animals, suggest that the carbohydrate-dependent energy deficiency exhibited by $A n \times A 6^{-/-}$mice induced higher lipid catabolism and reduced hepatic steatosis during the prereplicative phase of liver regeneration.

Besides triggering the regeneration program, the removal of $2 / 3$ of the liver mass induces an energetic/metabolic stress due to the drastic reduction of hepatic glycogen content and gluconeogenic capacity, both responsible for the rapid hypoglycaemia observed after surgery (3, 39). Several studies examining glucose supplementation in mice showed low hepatocellular proliferation in response to $\mathrm{PHx}(31,37,39)$, implicating the need to undergo hypoglycaemia to 
induce initial cell proliferation after hepatectomy. However, other studies discovered that continuous infusion of glucose before and after PHx prevented both the loss of glycogen and lipid deposition in rats, with no effect on DNA synthesis (36). AnxA6 ${ }^{-/-}$hepatectomised mice suffered rapid and prolonged hypoglycaemia immediately after surgery, while the priming phase and DNA synthesis evenly progressed during early stages of PHx. However, AnxA6 ${ }^{-/-}$mice were unable to recover from hypoglycaemia, causing death of almost $80 \%$ of AnxA6 ${ }^{-/-}$animals within 3 days, most likely due to the development of hypoglycaemic coma. Furthermore, the administration of glucose in the drinking water restored survival index of AnxA6 ${ }^{-/}$animals without avoiding initial hypoglycaemia after $\mathrm{PHx}$, clearly pointing to an energetic hindrance in hepatectomized AnxA6 $6^{-/-}$animals. Thirty percent of AnxA6 $6^{-/}$mice died during the first $24 \mathrm{~h}$ after $\mathrm{PHx}$, suggesting especially high glucose requirements of AnxA6 $6^{-/-}$mice at this stage. Although onset of hypoglycaemia after PHx is expected, supporting the triggering of alternative metabolic routes that fuel hepatic regeneration (31), the faster glycogen degradation and lower blood glucose levels in AnxA6 ${ }^{-/-}$mice compared to controls during the initial hours after PHx appear critical factors that contribute to determine AnxA6 $6^{-/-}$mice survival. Similarly, low survival rate in the fulminant hepatic failure (FHF) models is substantially rescued upon glucose administration (40) and inhibition of GNG in this FHF-disease model is well known to trigger hepatic encephalopathy (41).

The availability of gluconeogenic substrates in the liver determine the flux rates of GNG (4), and the amino acid transporters of the SNAT family are critical for the uptake of neutral amino acids such as alanine in the liver (42). The expression levels of both SNAT2 and SNAT4 are regulated by hormones, such as insulin, through the PI3K signalling pathway (7) and withdrawal of extracellular amino acids (43). Hepatic SNAT2 and SNAT4 expression also increased in diabetes-induced rodent models (44), under conditions when hormonal control of GNG is regulated. Nevertheless, despite upregulation of insulin levels in both WT and AnxA6 ${ }^{-/}$ animals after PHx, we only observed significantly increased hepatic SNAT4, but not SNAT2, mRNA levels in WT mice, indicating differential hormonal response of transcription factors regulating these SNATs that is compromised in AnxA6 ${ }^{-/-}$mice.

Multiple transport systems for the cellular exchange of amino acids are present in the plasma membrane of mammalian cells. This includes system A transporters, although very little is known about the machinery that regulates their subcellular localization. Interestingly, previous 
studies indicated SNAT2 redistribution to the hepatocyte plasma membrane after PHx (45). In skeletal muscle cells and adipocytes, the recruitment of SNAT2 to the cell surface from endosomal compartments or the trans-Golgi network appeared via insulin-signalling in a PKB/PI3K-dependent manner, possibly involving Syntaxin 6 (Stx6)-dependent exocytic trafficking events (46). Similarly, SNAT4 would be expected to follow the secretory pathway to be delivered to the blood-facing sinusoidal plasma membrane of hepatocytes. Our findings identify that in resting hepatocytes, most of this transporter was stored in a cytoplasmic endocytic compartment to be rapidly delivered to the sinusoidal plasma membrane after liver resection. Possibly related to these findings, we previously demonstrated AnxA6-induced changes in the cellular distribution of cholesterol to modulate exocytic membrane transport routes via several cholesterol-sensitive SNARE complexes, including Stx4, SNAP23 and Stx6 $(11,12)$. It is tempting to speculate that these AnxA6-induced changes in SNARE activity may contribute to SNAT4 mislocalisation after $\mathrm{PHx}$ in hepatocytes. Moreover, the multiple scaffolding functions of AnxA6 in several signalling pathways (13) may also contribute to control hormone-dependent SNAT4 translocation to the plasma membrane.

In addition to liver regeneration, regulation of SNAT activity could be also important in tumour pathogenesis. The single-nucleotide polymorphism rs2429467C $>$ T of SNAT4 has been associated with lung cancer (47), and the $292 \mathrm{C}>\mathrm{T}$ and $1304 \mathrm{G}>\mathrm{A}$ SNAT4 mutant alleles have been associated with hyperglycaemia in humans (48). Moreover, AnxA6 expression is downregulated in hepatocellular carcinoma (HCC) biopsies (49), and low SNAT4 as well as AnxA6 expression levels are associated with reduced survival in liver cancer patients (Cancer Genome Atlas (TGCA)). Furthermore, in a recent study, dysregulation of several GNG enzymes in HCC underlying macrovascular invasion was identified (50). Strikingly, in the same study, AnxA6, among a few others, was recognized to be a significantly downregulated biomarker protein in $\mathrm{HCC}$.

Altogether, our results link intracellular trafficking pathways with the regulation of glucose metabolism in the hepatocyte through AnxA6, a yet unrecognized/novel regulator of liver alanine transport and gluconeogenesis in mice. 


\section{ACKNOWLEDGEMENTS}

CR is supported by the Serra Húnter Programme (Generalitat de Catalunya). We are thankful to the staff from Centres Científics i Tecnològics (CCiT-UB), Universitat de Barcelona, Campus Casanova, Unitat de Microscòpia Òptica Avançada and Unitat de Microscòpia Electrònica. We are also grateful to Maria Molinos (University of Barcelona) for technical assistance.

\section{FIGURE LEGENDS}

Figure 1. AnxA6 is necessary for survival during liver regeneration in mice.

A. Survival index plot of WT (grey line, $n=23$ ) and AnxA6 ${ }^{-/-}$mice (orange line, $n=27$ ) after PHx (0-72 h). Log-rank (Mantel-Cox) test, $p<0.0001$.

B. Hepatic regeneration index at different time points $(0-72 \mathrm{~h})$ after PHx from WT $(\mathrm{n}=6-10$ per time point) and AnxA6 ${ }^{--}$(n=6-10 per time point) mice. Two-way ANOVA with Bonferroni post-hoc test, $p>0.05$.

C. Western blot and quantification $(n=3)$ of relative hepatic AnxA6 expression levels in WT mice after PHx (0-72 h).

D. Western blot of hepatic GFP and AnxA6 expression in WT and AnxA6 ${ }^{-/-}$mice 15 days after rAAV infection and western blot of liver, lung and kidney showing endogenous or recombinant AnxA6 expression in WT, AnxA6 ${ }^{-/-}$and $\mathrm{AAAV}-\mathrm{AnxA} 6$ infected AnxA6 ${ }^{-/-}$ $\left(\right.$ AnxA6 $6^{-/}+$rAAV-AnxA6) mice.

E. Survival index plot of WT+rAAV-GFP (grey line, $n=9$ ), AnxA6 ${ }^{-/}+$rAAV-GFP (orange line, $\mathrm{n}=9$ ) and AnxA6 ${ }^{-/}+\mathrm{rAAV}-\mathrm{AnxA6}$ mice (red line, n=9) after PHx (0-72 h). Log-rank (MantelCox) test, $p=0.032$.

F. Hepatic regeneration index from WT and AnxA6 ${ }^{-/-}$mice plus rAAV-GFP $(n=9)$ or rAAVAnxA6 (n=9) 7 days after PHx as indicated. Student $t$-test, $p>0.05$.

\section{Figure 2. Cell cycle progression in AnxA6 ${ }^{-/-}$mice after PHx.}

A. Quantification of Ki67 positive nuclei of 4 liver sections from WT $(n=6)$ and AnxA6 $6^{--}(n=6)$ mice $24 \mathrm{~h}$ after PHx. Student $t$-test, $p>0.05$. 
B-D. Relative mRNA expression levels of cJun (cJun), cFos (cFos) and Cyclin D1 (Ccndl) after PHx (0-6 h) in livers from WT and AnxA6-- mice (n=5). Two-way ANOVA with Bonferroni post-hoc test, $p>0.05$.

E. Representative PCNA-stained fields from WT and AnxA6 ${ }^{-/}$liver sections $48 \mathrm{~h}$ after PHx. Scale bar, $50 \mu \mathrm{m}$. Quantification of PCNA positive nuclei of 4 liver sections from WT ( $\mathrm{n}=6$ ) and $\mathrm{AnxA6}^{-/}$mice (n=7) $48 \mathrm{~h}$ after PHx. Student $t$-test, $p>0.05$.

F. Radioactive thymidine incorporation during liver regeneration $(0-72 \mathrm{~h})$ in WT $(\mathrm{n}=4-10)$ and AnxA6 $6^{-/}$mice $(\mathrm{n}=4-11)$. Two-way ANOVA with Bonferroni post-hoc test, $p>0.05$.

\section{Figure 3. Lipid accumulation in AnxA6 ${ }^{-/-}$livers after PHx.}

A. Representative H\&E staining of WT and AnxA6-/ liver sections 12 and $36 \mathrm{~h}$ after PHx. Scale bars, 50 and $20 \mu \mathrm{m}$.

B. Qualitative quantification of steatosis levels of liver sections from WT ( $\mathrm{n}=5-14)$ and AnxA6 ${ }^{-/}$ mice ( $\mathrm{n}=4-14)$ after PHx (0-72 h). Two-way ANOVA with Bonferroni post-hoc test, $p=0.0044$.

C. Quantification of hepatic TAG levels in WT $(n=4)$ and AnxA6-/ mice $(n=4)$ at 0,24 and $48 \mathrm{~h}$ after PHx. Two-way ANOVA with Bonferroni post-hoc test, $p=0.0001$.

D. Representative electron microscopy images of WT and AnxA6 ${ }^{-/-}$liver sections $0,12,36$ and $48 \mathrm{~h}$ after PHx. N, nucleus; *, lipid droplets; arrowheads, glycogen. Scale bar, $5 \mu \mathrm{m}$.

E. Quantification of blood ketone bodies in WT $(n=5)$ and AnxA6 ${ }^{-/}$mice $(n=5) 24$ and $48 \mathrm{~h}$ after PHx.

Figure 4. Blood glucose levels and glucose supplementation in AnxA6 ${ }^{-/-}$mice after PHx.

A. Blood glucose levels in WT ( $\mathrm{n}=6-10)$ and AnxA6 ${ }^{-/-}$mice $(\mathrm{n}=6-12)$ after PHx (0-72 h). Twoway ANOVA with Bonferroni post-hoc test, $p<0.0001$.

B. Plasma insulin levels in WT ( $\mathrm{n}=4-9)$ and AnxA6 ${ }^{-/}$mice ( $\left.\mathrm{n}=4-9\right)$ after PHx (0-48 h). Two-way ANOVA with Bonferroni post-hoc test, $p>0.05$.

C. Liver glycogen levels in WT $(\mathrm{n}=5)$ and $A n x A 6^{-/}$mice $(\mathrm{n}=5)$ after PHx (0-12 h). Two-way ANOVA with Bonferroni post-hoc test, $p=0.0045$.

D. Survival index plot of WT (grey line, $n=7$ ) and AnxA6 $6^{-/-}$mice (orange line, $n=11$ ) after PHx $(0-72$ h) with $10 \%$ glucose supplemented drinking water. Log-rank (Mantel-Cox) test, 
$p=0.705$. Survival index of WT (grey dotted line) and AnxA6 $6^{-/-}$mice (orange dotted line) after PHx is also shown.

E. Hepatic regeneration index from WT $(n=7)$ and AnxA6-/- mice $(n=9) 7$ days after PHx with $10 \%$ glucose supplemented drinking water. Student $t$-test, $p>0.05$.

F. Blood glucose levels in WT (n=9-10) and AnxA6 $6^{-/}$mice $(n=7-10)$ after PHx (0-72 h) with $10 \%$ glucose supplemented drinking water. Two-way ANOVA with Bonferroni post-hoc test, $p<0.0001$.

Figure 5. AnxA6 deficiency impairs alanine-dependent hepatic gluconeogenesis after PHx.

A. Glucose secretion of WT and AnxA6 ${ }^{-/-}$primary isolated hepatocytes $(n=4)$ starved for $6 \mathrm{~h}$ and incubated with $2 \mathrm{mM}$ sodium pyruvate, $20 \mathrm{mM}$ L-alanine and $20 \mathrm{mM} \mathrm{L}$-glutamine.

B. L-alanine plasma levels in WT and AnxA6 ${ }^{-/}$mice after PHx (0-48 h, $\mathrm{n}=4$ each group). Twoway ANOVA with Bonferroni post-hoc test, $p=0.0097$.

C. Total amino acid plasma levels in WT and AnxA6 ${ }^{-/-}$mice after PHx (0-48 h, $\mathrm{n}=4$ each group). Two-way ANOVA with Bonferroni post-hoc test, $p>0.05$.

D. L-alanine hepatic levels in WT and AnxA6 ${ }^{-/ 2}$ mice after PHx (0-48 h, $\mathrm{n}=4$ each group). Twoway ANOVA with Bonferroni post-hoc test, $p=0.0404$.

E. Total amino acid hepatic levels in WT and AnxA6 ${ }^{-/-}$mice after PHx (0-48 h, n=4 each group). Two-way ANOVA with Bonferroni post-hoc test, $p>0.05$.

Figure 6. Alanine deamination and Pck1 expression in livers of AnxA6 ${ }^{-/-}$mice after PHx.

A-B. Relative mRNA expression levels of ALAT1 (Gpt1) and ALAT2 (Gpt2) after PHx in WT and AnxA6 $6^{-/-}$liver (0-48 h, $\mathrm{n}=5$ each group). Two-way ANOVA with Bonferroni post-hoc test, $p<0.0001(\mathrm{C})$.

C. Hepatic alanine aminotransferase (ALAT) activity after PHx in WT and AnxA6 ${ }^{-/ 2}$ mice (0-48 $\mathrm{h}, \mathrm{n}=5$ each group). Two-way ANOVA with Bonferroni post-hoc test, $p>0.05$.

D. Relative mRNA expression levels of PEPCK (Pckl) after PHx in WT and AnxA6 $6^{-/-}$liver (0$48 \mathrm{~h}, \mathrm{n}=5$ each group). Two-way ANOVA with Bonferroni post-hoc test, $p=0.0011$. 
Figure 7. AnxA6 $6^{-/-}$deficiency reduces alanine uptake in isolated hepatocytes and is associated with SNAT4 mislocalisation in livers after PHx.

A. Radioactive $\left[{ }^{14} \mathrm{C}\right]$-L-alanine uptake of WT and AnxA6 ${ }^{-/-}$primary hepatocytes $(0-10 \mathrm{~min}, \mathrm{n}=4)$. Two-way ANOVA test, $p=.001$.

B-C. Relative mRNA expression levels of SNAT2 (Slc38a2) and SNAT4 (Slc38a4) after PHx in WT and AnxA6 $6^{-/-}$liver (0-48 h, n=5 each group). Two-way ANOVA with Bonferroni posthoc test, $p>0.05(\mathrm{~B})$ and $p<0.0028(\mathrm{C})$.

D. Relative SNAT4 protein expression in WT and AnxA6 ${ }^{-/-}$mice $48 \mathrm{~h}$ after PHx ( $\mathrm{n}=4$ each group). Student $t$-test, $p>0.05$.

E. Representative confocal laser scanning microscopy of WT and AnxA6 $6^{-/-}$liver sections at 0 , 12, 24 and $48 \mathrm{~h}$ after PHx stained for SNAT4 (green), phalloidin (actin, red) and DAPI (nucleus, blue). Scale bar, $10 \mu \mathrm{m}$.

F. Quantification of SNAT4 intensity ratio between sinusoidal plasma membrane and cytoplasm intensities of WT and AnxA6 $6^{-/-}$liver sections ( $\mathrm{n}=4$ each group) at $0,12,24$ and $48 \mathrm{~h}$ after PHx. Two-way ANOVA with Bonferroni post-hoc test, $p<0.0001$.

Figure 8. Model of AnxA6-mediated and SNAT4-dependent regulation of gluconeogenesis in hepatocytes $48 \mathrm{~h}$ after PHx.

Schematic representation of SNAT4-dependent alanine uptake and gluconeogenesis in WT and $\mathrm{AnxA6}^{-/}$hepatocytes. The lack of SNAT4 recycling to the sinusoidal membrane in AnxA6 ${ }^{-/}$ mice $48 \mathrm{~h}$ after PHx strongly hinders alanine uptake and compromises alanine-dependent gluconeogenesis required for liver regeneration.

\section{REFERENCES}

1. Riehle KJ, Dan YY, Campbell JS, Fausto N. New concepts in liver regeneration. J Gastroenterol Hepatol 2011;26 Suppl 1:203-212.

2. Michalopoulos GK. Principles of liver regeneration and growth homeostasis. Compr Physiol 2013;3:485-513. 
3. Weymann A, Hartman E, Gazit V, Wang C, Glauber M, Turmelle Y, Rudnick DA. p21 is required for dextrose-mediated inhibition of mouse liver regeneration. Hepatology 2009;50:207215.

4. Rui L. Energy metabolism in the liver. Compr Physiol 2014;4:177-197.

5. Klain GJ, Winders RL, Bonner SJ. Sequential changes in alanine metabolism following partial hepatectomy in the rat. J Nutr Biochem 1990;1:578-584.

6. Mackenzie B, Erickson JD. Sodium-coupled neutral amino acid (System N/A) transporters of the SLC38 gene family. Pflugers Arch 2004;447:784-795.

7. Gu S, Langlais P, Liu F, Jiang JX. Mouse system-N amino acid transporter, mNAT3, expressed in hepatocytes and regulated by insulin-activated and phosphoinositide 3-kinasedependent signalling. Biochem J 2003;371:721-731.

8. Kondou H, Kawai M, Tachikawa K, Kimoto A, Yamagata M, Koinuma T, Yamazaki M, et al. Sodium-coupled neutral amino acid transporter 4 functions as a regulator of protein synthesis during liver development. Hepatol Res 2013;43:1211-1223.

9. Enrich C, Rentero C, Grewal T. Annexin A6 in the liver: From the endocytic compartment to cellular physiology. Biochim Biophys Acta Mol Cell Res 2017;1864:933-946.

10. Enrich C, Rentero C, de Muga SV, Reverter M, Mulay V, Wood P, Koese M, et al. Annexin A6-Linking $\mathrm{Ca}(2+)$ signaling with cholesterol transport. Biochim Biophys Acta 2011;1813:935-947.

11. Reverter M, Rentero C, de Muga SV, Alvarez-Guaita A, Mulay V, Cairns R, Wood P, et al. Cholesterol transport from late endosomes to the Golgi regulates t-SNARE trafficking, assembly, and function. Mol Biol Cell 2011;22:4108-4123.

12. Garcia-Melero A, Reverter M, Hoque M, Meneses-Salas E, Koese M, Conway JR, Johnsen $\mathrm{CH}$, et al. Annexin A6 and Late Endosomal Cholesterol Modulate Integrin Recycling and Cell Migration. J Biol Chem 2016;291:1320-1335.

13. Grewal T, Koese M, Rentero C, Enrich C. Annexin A6-regulator of the EGFR/Ras signalling pathway and cholesterol homeostasis. Int J Biochem Cell Biol 2010;42:580-584.

14. Alvarez-Guaita A, Vila de Muga S, Owen DM, Williamson D, Magenau A, GarciaMelero A, Reverter M, et al. Evidence for annexin A6-dependent plasma membrane remodelling of lipid domains. Br J Pharmacol 2015;172:1677-1690. 
15. Tagoe CE, Boustead CM, Higgins SJ, Walker JH. Characterization and immunolocalization of rat liver annexin VI. Biochim Biophys Acta 1994;1192:272-280.

16. Hawkins TE, Roes J, Rees D, Monkhouse J, Moss SE. Immunological development and cardiovascular function are normal in annexin VI null mutant mice. Mol Cell Biol 1999;19:80288032.

17. Song G, Harding SE, Duchen MR, Tunwell R, O'Gara P, Hawkins TE, Moss SE. Altered mechanical properties and intracellular calcium signaling in cardiomyocytes from annexin 6 null-mutant mice. FASEB J 2002;16:622-624.

18. Krautbauer S, Haberl EM, Eisinger K, Pohl R, Rein-Fischboeck L, Rentero C, AlvarezGuaita A, et al. Annexin A6 regulates adipocyte lipid storage and adiponectin release. Mol Cell Endocrinol 2017;439:419-430.

19. Cairns R, Fischer AW, Blanco-Munoz P, Alvarez-Guaita A, Meneses-Salas E, Egert A, Buechler C, et al. Altered hepatic glucose homeostasis in AnxA6-KO mice fed a high-fat diet. PLoS One 2018;13:e0201310.

20. Higgins GM, Anderson RM. Experimental pathology of the liver. I. Restoration of the liver of the white rat following partial surgical removal. Arch. Pathol. 1931;12:186-202.

21. Zou CG, Gao SY, Zhao YS, Li SD, Cao XZ, Zhang Y, Zhang KQ. Homocysteine enhances cell proliferation in hepatic myofibroblastic stellate cells. J Mol Med (Berl) 2009;87:75-84.

22. Assy N, Minuk GY. Liver regeneration: methods for monitoring and their applications. J Hepatol 1997;26:945-952.

23. Fernandez MA, Albor C, Ingelmo-Torres M, Nixon SJ, Ferguson C, Kurzchalia T, Tebar F, et al. Caveolin-1 is essential for liver regeneration. Science 2006;313:1628-1632.

24. Leffert HL, Koch KS, Moran T, Rubalcava B. Hormonal control of rat liver regeneration. Gastroenterology 1979;76:1470-1482.

25. Huang J, Rudnick DA. Elucidating the metabolic regulation of liver regeneration. Am J Pathol 2014;184:309-321.

26. Naslavsky N, Caplan S. The enigmatic endosome - sorting the ins and outs of endocytic trafficking. J Cell Sci 2018;131.

27. Broer S. The SLC38 family of sodium-amino acid co-transporters. Pflugers Arch 2014;466:155-172. 
28. Blindenbacher A, Wang X, Langer I, Savino R, Terracciano L, Heim MH. Interleukin 6 is important for survival after partial hepatectomy in mice. Hepatology 2003;38:674-682.

29. Kong B, Huang J, Zhu Y, Li G, Williams J, Shen S, Aleksunes LM, et al. Fibroblast growth factor 15 deficiency impairs liver regeneration in mice. Am J Physiol Gastrointest Liver Physiol 2014;306:G893-902.

30. Nakatani T, Ozawa K, Asano M, Ukikusa M, Kamiyama Y, Tobe T. Changes in predominant energy substrate after hepatectomy. Life Sci 1981;28:257-264.

31. Rudnick DA, Davidson NO. Functional Relationships between Lipid Metabolism and Liver Regeneration. Int J Hepatol 2012;2012:549241.

32. Walldorf J, Hillebrand C, Aurich H, Stock P, Hempel M, Ebensing S, Fleig WE, et al. Propranolol impairs liver regeneration after partial hepatectomy in $\mathrm{C} 57 \mathrm{Bl} / 6$-mice by transient attenuation of hepatic lipid accumulation and increased apoptosis. Scand J Gastroenterol 2010;45:468-476.

33. Gazit V, Weymann A, Hartman E, Finck BN, Hruz PW, Tzekov A, Rudnick DA. Liver regeneration is impaired in lipodystrophic fatty liver dystrophy mice. Hepatology 2010;52:21092117.

34. Newberry EP, Kennedy SM, Xie Y, Luo J, Stanley SE, Semenkovich CF, Crooke RM, et al. Altered hepatic triglyceride content after partial hepatectomy without impaired liver regeneration in multiple murine genetic models. Hepatology 2008;48:1097-1105.

35. Shteyer E, Liao Y, Muglia LJ, Hruz PW, Rudnick DA. Disruption of hepatic adipogenesis is associated with impaired liver regeneration in mice. Hepatology 2004;40:13221332.

36. Simek JF, Rubin F, Lieberman I. Synthesis of DNA after partial hepatectomy without changes in the lipid and glycogen contents of the liver. Biochem Biophys Res Commun 1968;30:571-575.

37. Caruana JA, Whalen DA, Jr., Anthony WP, Sunby CR, Ciechoski MP. Paradoxical effects of glucose feeding on liver regeneration and survival after partial hepatectomy. Endocr Res 1986;12:147-156.

38. Lai HS, Chen WJ, Chen KM. Energy substrate for liver regeneration after partial hepatectomy in rats: effects of glucose vs fat. JPEN J Parenter Enteral Nutr 1992;16:152-156. 
39. Huang J, Schriefer AE, Cliften PF, Dietzen D, Kulkarni S, Sing S, Monga SP, et al. Postponing the Hypoglycemic Response to Partial Hepatectomy Delays Mouse Liver Regeneration. Am J Pathol 2016;186:587-599.

40. Eguchi S, Kamlot A, Ljubimova J, Hewitt WR, Lebow LT, Demetriou AA, Rozga J. Fulminant hepatic failure in rats: survival and effect on blood chemistry and liver regeneration. Hepatology 1996;24:1452-1459.

41. Dabos KJ, Whalen HR, Newsome PN, Parkinson JA, Henderson NC, Sadler IH, Hayes PC, et al. Impaired gluconeogenesis in a porcine model of paracetamol induced acute liver failure. World J Gastroenterol 2011;17:1457-1461.

42. Freeman TL, Ngo HQ, Mailliard ME. Inhibition of system A amino acid transport and hepatocyte proliferation following partial hepatectomy in the rat. Hepatology 1999;30:437-444.

43. Hoffmann TM, Cwiklinski E, Shah DS, Stretton C, Hyde R, Taylor PM, Hundal HS. Effects of Sodium and Amino Acid Substrate Availability upon the Expression and Stability of the SNAT2 (SLC38A2) Amino Acid Transporter. Front Pharmacol 2018;9:63.

44. Varoqui H, Erickson JD. Selective up-regulation of system a transporter mRNA in diabetic liver. Biochem Biophys Res Commun 2002;290:903-908.

45. Freeman TL, Thiele GM, Tuma DJ, Machu TK, Mailliard ME. ATA2-mediated amino acid uptake following partial hepatectomy is regulated by redistribution to the plasma membrane. Arch Biochem Biophys 2002;400:215-222.

46. Hatanaka T, Hatanaka Y, Tsuchida J, Ganapathy V, Setou M. Amino acid transporter ATA2 is stored at the trans-Golgi network and released by insulin stimulus in adipocytes. J Biol Chem 2006;281:39273-39284.

47. Lee SY, Kang HG, Choi JE, Jung DK, Lee WK, Lee HC, Lee SY, et al. Polymorphisms in cancer-related pathway genes and lung cancer. Eur Respir J 2016;48:1184-1191.

48. Gonzalez-Renteria SM, Loera-Castaneda V, Chairez-Hernandez I, Sosa-Macias M, Paniagua-Castro N, Lares-Aseff I, Rodriguez-Moran M, et al. Association of the polymorphisms $292 \mathrm{C}>\mathrm{T}$ and $1304 \mathrm{G}>\mathrm{A}$ in the SLC38A4 gene with hyperglycaemia. Diabetes Metab Res Rev 2013;29:39-43.

49. Meier EM, Rein-Fischboeck L, Pohl R, Wanninger J, Hoy AJ, Grewal T, Eisinger K, et al. Annexin A6 protein is downregulated in human hepatocellular carcinoma. Mol Cell Biochem 2016;418:81-90. 
50. Cao Y, Ding W, Zhang J, Gao Q, Yang H, Cao W, Wang Z, et al. Significant DownRegulation of Urea Cycle Generates Clinically Relevant Proteomic Signature in Hepatocellular Carcinoma Patients with Macrovascular Invasion. J Proteome Res 2019;18:2032-2044. 